\title{
Mesothelial Cyst of the Round Ligament Mimicking a Metastasis: a Case Report
}

\author{
Bo Mi Kim, MD' \\ Ji Young Lee, MD ${ }^{1}$ \\ Yoon Hee Han, MD' \\ Su Young Kim, MD' \\ Jung Wook Seo, MD' \\ Yong Hoon Kim, MD' \\ Soon Joo Cha, MD ${ }^{1}$ \\ Gham Hur, MD ${ }^{1}$ \\ Mee Joo, MD² \\ Eung Soo Lee, MD
}

\section{Index terms :}

Mesothelial cyst

Round ligament

Inguinal area

Ultrasonography

Multi-detector row CT

DOI:10.3348/kjr.2010.11.3.364

Korean J Radiol 2010; $11: 364-367$

Received July 24, 2009; accepted

after revision October 26, 2009.

Departments of ${ }^{1}$ Radiology, ${ }^{2}$ Pathology, ${ }^{3}$ Obstetrics and Gynecology, Ilsan Paik Hospital, Inje University, School of

Medicine, Gyeonggi-do 411-706, Korea

Address reprint requests to:

Ji Young Lee, MD, Department of

Radiology, Ilsan Paik Hospital, Inje

University, School of Medicine, 2240,

Daehwa-dong, Ilsan-gu, Goyang-si,

Gyeonggi-do 411-706, Korea.

Tel. (8231) 910-7689

Fax. (8231) 910-7369

e-mail: drleeji@paik.ac.kr
A mesothelial cyst of the round ligament is a rare cause of an inguinal mass. Clinically, it is frequently misdiagnosed as one of commoner diseases such as an inguinal hernia, femoral hernia, lipoma, and lymphadenopathy upon physical examination. Some previous reports elaborated the sonographic features of a mesothelial cyst of the round ligament. However, to our knowledge, few reports have described the CT features of a mesothelial cyst. We illustrated here the sonographic and multidetector CT features of a case of a mesothelial cyst of the round ligament that presented as an inguinal palpable mass and mimicked a metastasis in a patient with a Sertoli-Leydig cell tumor of the ovary.

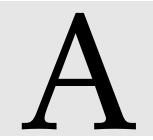
mesothelial cyst of the round ligament is a rare developmental disorder, which is often associated with and misdiagnosed as an inguinal hernia (1). A mesothelial cyst is not usually considered in the differential diagnoses of inguinal masses in women, and is little-known radiologically. Previous studies exist on mesothelial cysts; however, these focus on their sonographic features. To the best of our knowledge, only one case have described the CT feature of a mesothelial cyst (2). Herein, we present the sonographic and multidetector CT (MDCT) features of a mesothelial cyst of the round ligament, which presented as an inguinal mass in a patient with a Sertoli-Leydig cell tumor of the ovary, and mimicked a metastasis of the round ligament. We also discuss the differential points between a mesothelial cyst and other masses of the round ligament.

\section{CASE REPORT}

A 76-year-old woman visited our gynecologic department with masses in her lower abdomen and left inguinal region for seven months. She also had experienced intermittent vaginal spotting over the last three years. Upon physical examination, a large, hard abdominal mass that extended from the pelvis to $7 \mathrm{~cm}$ above the umbilicus was noted. In addition, a tense, painless mass $5 \mathrm{~cm}$ in size was detected in her left inguinal region. The inguinal mass was not reduced by manual pressure in the supine position, nor was it changed in size by Valsalva's maneuver. Also, no evidence of inflammation or incarceration was noted. The serum cancer antigen 125 level was elevated to $106.0 \mathrm{U} / \mathrm{mL}$ (normal range, $<35 \mathrm{U} / \mathrm{mL}$ ), and the white blood cell counts were within the normal limit.

Transabdominal ultrasonography revealed a huge, $21 \times 12 \times 10 \mathrm{~cm}$, solid and cystic mass with increased blood flow within the solid and septal parts of the abdominal cavity. High-resolution ultrasonography with a $12-5 \mathrm{MHz}$ linear-array transducer showed a well-circumscribed, oval cystic mass with a hypoechoic solid portion $(3 \times 3 \times$ 

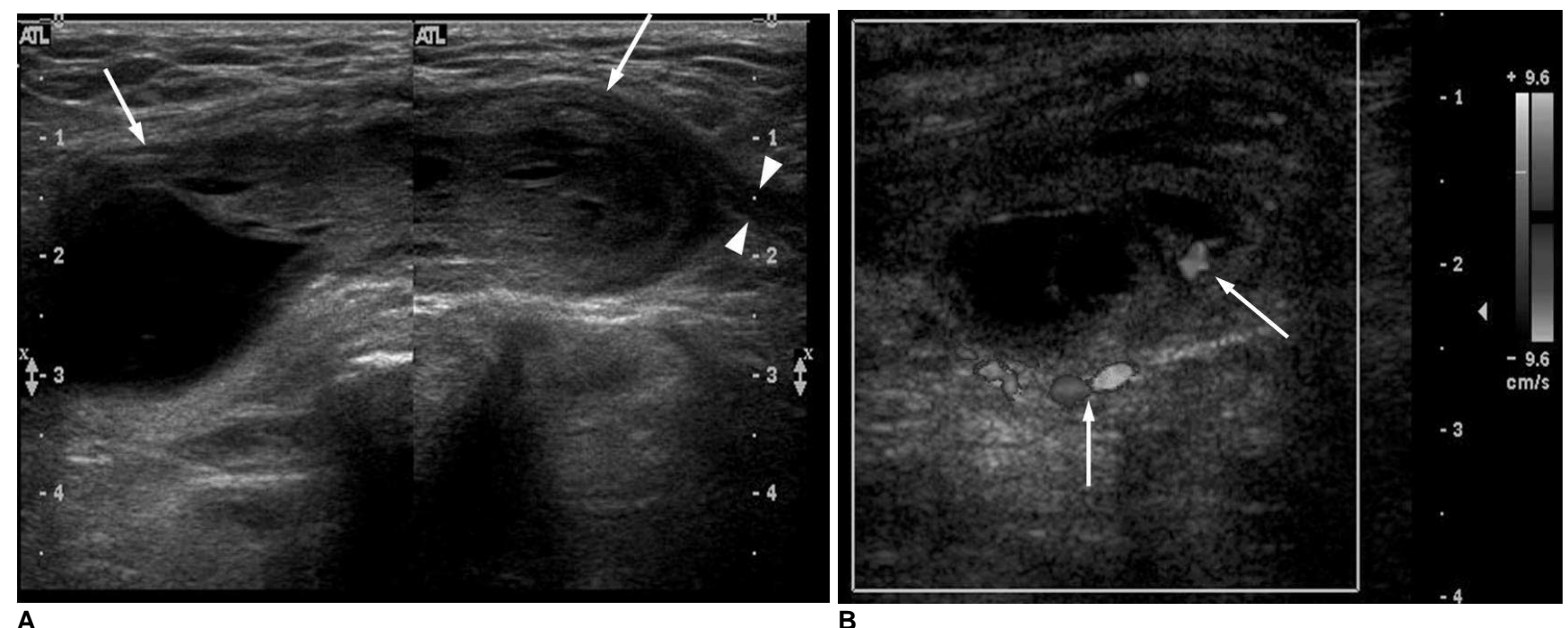

A
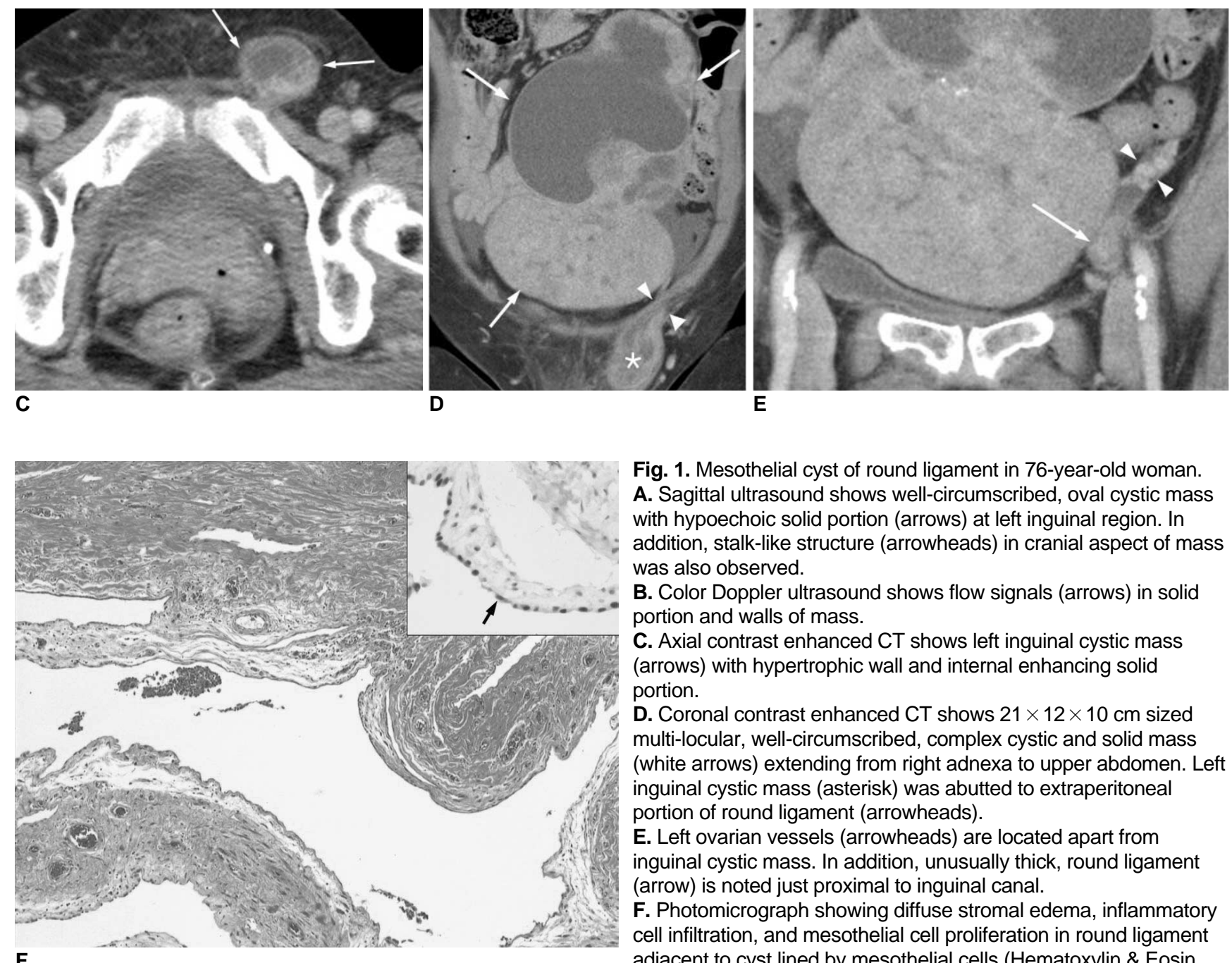

Fig. 1. Mesothelial cyst of round ligament in 76-year-old woman. A. Sagittal ultrasound shows well-circumscribed, oval cystic mass with hypoechoic solid portion (arrows) at left inguinal region. In addition, stalk-like structure (arrowheads) in cranial aspect of mass was also observed.

B. Color Doppler ultrasound shows flow signals (arrows) in solid portion and walls of mass.

C. Axial contrast enhanced CT shows left inguinal cystic mass (arrows) with hypertrophic wall and internal enhancing solid portion.

D. Coronal contrast enhanced CT shows $21 \times 12 \times 10 \mathrm{~cm}$ sized multi-locular, well-circumscribed, complex cystic and solid mass (white arrows) extending from right adnexa to upper abdomen. Left inguinal cystic mass (asterisk) was abutted to extraperitoneal portion of round ligament (arrowheads).

E. Left ovarian vessels (arrowheads) are located apart from inguinal cystic mass. In addition, unusually thick, round ligament (arrow) is noted just proximal to inguinal canal.

F. Photomicrograph showing diffuse stromal edema, inflammatory cell infiltration, and mesothelial cell proliferation in round ligament adjacent to cyst lined by mesothelial cells (Hematoxylin \& Eosin staining, $\times 100)$. Immunostaining of WT-1 and mesothelial cell marker highlight cyst-lining mesothelial cells (arrow) (inset, $\times 200$ ). 


\section{Kim et al.}

$6 \mathrm{~cm}$ ) in the left inguinal region. Also, a stalk-like structure was seen in the cranial aspect of the mass (Fig. 1A). The mass did not show peristalsis or any bowel content. Color Doppler sonography showed flow signals in the solid portion and in the wall of the mass (Fig. 1B).

The contrast-enhanced MDCT with multi-planar reformatted images showed the large abdominal mass arising from the right adnexa and extending to the upper abdominal cavity. The left inguinal cystic mass was abutted to the extraperitoneal portion of the round ligament, which was unusually thickened, and had an irregular enhancing solid portion in the wall of the cystic mass. The presumed preoperative diagnosis was a malignant right ovarian mass with metastasis in the left round ligament. Herniation of the left ovary cases were excluded from the patient population because the reformatted $\mathrm{CT}$ images revealed more cranially located ovarian vessels within the pelvic cavity (Figs. 1C-E).

At surgery, the large mass originated from the right ovary, and the left ovary was atrophied. A cystic mass in the inguinal region was attached to the hypertrophic left round ligament.

Upon histopathologic examination, the right ovarian mass was confirmed as a Sertoli-Leydig cell tumor. The inguinal mass was a multi-locular cyst lined by mesothelial cells, and combined with diffuse stromal edema and chronic inflammation (Fig. 1F). However, no evidence of malignancy was observed.

\section{DISCUSSION}

The round ligament extends from the uterus, through the inguinal canal, and ends in the region of the mons pubis and labia major. Embryologically, this is the female equivalent of gubernaculums testis and is predominantly composed of smooth muscle fibers, connective tissue, vessels, and nerves with a mesothelial coating (3). There have been two theories suggested for the development of a mesothelial cyst of the round ligament. The first one is a flawed obliteration of Nuck's canal. In males, a comparable flaw in the obliteration may result in a cystic hydrocele or a hydrocele of the spermatic cord. According to this theory, a round ligament cyst is the same disease as a cyst of the Nuck's canal. The other one is the inclusion of embryonic mesenchymal mesothelial elements or remnants during the development of the round ligament $(1,4)$. Cysts of mesothelial investment of the round ligament are lined with a single layer of flat, cuboidal cells that have the characteristic appearance of mesothelial cells. The round ligament cyst and Nuck's canal cyst ultimately show the same imaging and histologic features (4).
Ultrasonography is the imaging modality of choice for the inguinal mass due to the easy application and availability of real-time information about the peristalsis or changes by coughing or Valsalva's maneuver. Round ligament cysts have been described as having various sonographic appearances on previous reports including: a dumbbellshaped cyst with the internal septum, comma- or fusiform, oval cystic mass with a connection to the peritoneal cavity or inguinal canal, and lastly a cyst within a mass appearing to be a cyst without a connection to abdominal cavity (57). The sonographic findings of our case were similar to previous reports, which cite an oval cystic mass with a stalk-like structure that connected at the inguinal canal. However, the findings of internal hypoechoic solid portions with an eccentric thickened wall were unusual.

Multidetector CT scan showed a cystic mass with an irregular wall thickening and enhancement. This finding led to the misdiagnosis as a metastatic lesion in the round ligament. Histologically the results indicate a diffuse stromal edema and chronic inflammatory cell infiltration. The optimal vascular enhancement on MDCT excluded the herniation of the left ovary by showing ovarian vessels, which was locationally separate from the round ligament and the inguinal cystic mass. To date, there has been only one report including the $\mathrm{CT}$ findings of a mesothelial cyst as a lobulated cystic mass located in the inguinal region on an axial CT scan (2). MDCT with a multi-planar reformatted image can provide superior anatomic orientation of the mass arising from the round ligament and can be helpful for the differential diagnoses of the various masses in the inguinal region.

Clinically, a mesothelial cyst of the round ligament is usually misdiagnosed as an inguinal hernia because of its anatomic location and relatively rare occurrence.

Furthermore, a mesothelial cyst may be associated with an inguinal hernia in about $30-40 \%$ of cases $(5,8)$. However, since we could not detect any bowel content in the mass by real time preoperative sonography, it was clear that an inguinal hernia could be excluded from the possible diagnoses. Other unusual tumors involving the round ligament have been reported in the literature: endometriosis, leiomyoma and in rare cases, a leiomyosarcoma (3). Leiomyoma, the most common lesion involving the round ligament, presents a mainly solid enhancing mass. For this reason, we could distinguish it from the mesothelial cyst (3). Endometriosis is typically seen as a homogeneous hypoechoic focal lesion with diffuse low-level internal echoes on ultrasonography (9). However, considering the finding of Yang et al. (10) indicating that inguinal endometriosis also could be seen as a multicystic mass without an internal echo, this sonographic feature may not 


\section{Mesothelial Cyst of Round Ligament Mimicking Metastasis}

be helpful for differentiation.

In conclusion, although rare, a mesothelial cyst of the extraperitoneal round ligament should be included in the differential diagnosis of an inguinal mass in female patients. Moreover, radiologists should be familiar with its feature in imaging, as well as be aware that it may present as a cystic mass with an enhancing solid portion on ultrasonography and CT scan.

\section{References}

1. Harper GB Jr, Awbrey BJ, Thomas CG Jr, Askin FB. Mesothelial cysts of the round ligament simulating inguinal hernia. Report of four cases and a review of the literature. Am J Surg 1986;151:515-517

2. Oh SN, Jung SE, Rha SE, Lim GY, Ku YM, Byun JY, et al. Sonography of various cystic masses of the female groin. $J$ Ultrasound Med 2007;26:1735-1742

3. Warshauer DM, Mandel SR. Leiomyoma of the extraperitoneal round ligament: CT demonstration. Clin Imaging 1999;23:375376
4. Oh SN, Jung SE, Lee JM, Chung JH, Park GS. Sonographic diagnosis of a round ligament cyst in the inguinal area. J Clin Ultrasound 2007;35:226-228

5. Khanna PC, Ponsky T, Zagol B, Lukish JR, Markle BM. Sonographic appearance of canal of Nuck hydrocele. Pediatr Radiol 2007;37:603-606

6. Park SJ, Lee HK, Hong HS, Kim HC, Kim DH, Park JS, et al. Hydrocele of the canal of Nuck in a girl: ultrasound and MR appearance. Br J Radiol 2004;77:243-244

7. Stickel WH, Manner M. Female hydrocele (cyst of the canal of Nuck): sonographic appearance of a rare and little-known disorder. J Ultrasound Med 2004;23:429-432

8. Ryley DA, Moorman DW, Hecht JL, Alper MM. A mesothelial cyst of the round ligament presenting as an inguinal hernia after gonadotropin stimulation for in vitro fertilization. Fertil Steril 2004;82:944-946

9. Cervini P, Mahoney J, Wu L. Endometriosis in the canal of Nuck: atypical manifestations in an unusual location. AJR Am J Roentgenol 2005;185:284-285

10. Yang DM, Kim HC, Jin W, Ryu CW, Ryu JK, Nam DH, et al. Inguinal endometriosis presenting as a multicystic mass on sonography. J Ultrasound Med 2007;26:1449-1451 